\title{
Reduced Rates of Metribuzin and Time of Hilling Controlled Weeds in Potato
}

\author{
Walaa Siblani, Mustapha A. Haidar* \\ Department of Agriculture, Faculty of Agriculture and Food Sciences, American University of Beirut, Beirut, Lebanon \\ Email: *mhaidar@aub.edu.lb
}

How to cite this paper: Siblani, W. and Haidar, M.A. (2017) Reduced Rates of Metribuzin and Time of Hilling Controlled Weeds in Potato. American Journal of Plant Sciences, 8, 3207-3217. https://doi.org/10.4236/ajps.2017.812216

Received: October 25, 2017

Accepted: November 25, 2017

Published: November 28, 2017

Copyright @ 2017 by authors and Scientific Research Publishing Inc. This work is licensed under the Creative Commons Attribution International License (CC BY 4.0).

http://creativecommons.org/licenses/by/4.0/

(c) (i) Open Access

\begin{abstract}
The current emphasis on reducing herbicide applications has led to an increase in alternative weed control measures. Field experiment was conducted in the spring of 2014 to examine the effect of hilling-time and reduced-rates of metribuzin and their combinations on weed infestation in potato, and to determine their impact on potato yield. Metribuzin at $0.35,0.56$, or $0.75 \mathrm{~kg}$ ai $/ \mathrm{ha}$ with or without hilling 6,7 , and 8 weeks after planting (WAP) were used. Weed count, weed control visual rating, weed dry weight, potato plant height, number of shoots and leaves, root dry weight, and potato yield were collected. Results showed that metribuzin, at all tested rates, with or without hilling significantly reduced weed infestation after 50, 70, and 110 days after planting (DAP) compared to the check. Best results were obtained by a combination of metribuzin at all tested rates with hilling 6,7 , and $8 \mathrm{WAP}$. The results suggest that long season weed control and high marketable yield could be achieved by metribuzin at $0.35 \mathrm{~kg}$ ai $/ \mathrm{ha}$ ( $53 \%$ reduction in metribuzin) supplemented with hilling (6 WAP). None of the treatments was toxic to potato plants compared to the hand-weeded plots.
\end{abstract}

\section{Keywords}

Potato, Metribuzin, Hilling, Weed Control

\section{Introduction}

Solanum tuberosum, commonly known as potato is considered one of the most important strategic crops in Lebanon and the Mediterranean region [1]. Potato is susceptible to several pests among which are weeds that compete for resources with summer, spring, and autumn planted potatoes across Lebanon and the Mediterranean region.

Weeds are major problem in potato production in Lebanon. They can cause 
significant loss of yield quality and quantity through direct competition for light, moisture, and nutrients, as well as harbor insects and diseases that attack potato. They also present a problem at harvest by increasing mechanical damage to tubers, reducing harvesting efficiency, and slowing down harvesting operations. In Lebanon, potato production involves using conventional tillage method, mechanical planting, and hilling within one month from planting. Hilling is accomplished mainly with a locally manufactured plow to aerate the soil, enhances tuber development, and prevents exposure of tubers to sunlight. While, weed management involves hand weeding and the use of pre or post application of herbicide Metribuzin $\left(\right.$ Sencor $^{\circledR}$ ) at $0.75 \mathrm{~kg}$ ai/ha. However, the globally rising public concern about the use of herbicides has shifted trends towards reduction in their use. The reliance on herbicides poses environmental and economic threat, since herbicides are expensive and can leach in the soil contaminating groundwater, especially when farmers apply high dosage to achieve maximum control instead of just satisfactory management [2]; Some weeds are becoming resistant to herbicides [3] [4]. Therefore, many researchers are investigating the benefits of integrated mechanical and herbicide techniques for weed management practices [5] [6] [7].

In order to reduce chemical load on the environment without significant loss in yield, the time and number of hilling operations and herbicide application rates must be optimized. Excessive tillage is costly and can increase soil compaction and lower tuber production, while herbicides pose a potential hazard to the environment. Taking into consideration the environmental and economic aspects posed by these practices, proper hilling times and herbicide rates should be maintained. Accordingly, the objective of this study was to examine weed control with, and potato tolerance to, various combinations of hilling-time and reduced-rates of metribuzin.

\section{Materials and Methods}

\subsection{Site Information}

The experiment was conducted on a $1275 \mathrm{~m}^{2}$ field at Advancing Research Enabling Communities Center (AREC), during April to September 2014. AREC is located in the Central Beq'aa plain with an altitude of around $1000 \mathrm{~m}$ above sea level at $34^{\circ} 54^{\prime \prime} \mathrm{N}$ latitude and $36^{\circ} 45^{\prime \prime} \mathrm{E}$ longitude. The soil is clayey ( $48.08 \%$ clay, $35.85 \%$ silt, and $15.92 \%$ sand $)$, basic $(\mathrm{pH}=7.80)$, non-saline $(\mathrm{EC}=0.00409$ $\mathrm{dS} / \mathrm{m}$ ), with $2.15 \%$ organic matter, $0.79 \% \mathrm{~N}, 16.9 \mathrm{ppm} \mathrm{P}, 415 \mathrm{ppm} \mathrm{K}$, and $37.33 \% \mathrm{CaCO}_{3}$. Soil analysis was done according to [8].

\subsection{General Experimental Procedures}

The experimental field was tilled twice with a conventional moldboard plow, disked, and leveled two weeks prior to potato planting. The experimental area received a uniform application of $200 \mathrm{~kg}$ of NPK (15:15:15) fertilizer one hour prior to potato sowing by a spreader, followed by shallow tillage. Another $50 \mathrm{~kg}$ 
of NPK was band applied during potato sowing. Four hundred kilograms of small standard potato cultivar "Spunta" tubers (20 tubers/row) were planted on April 17, 2014 in the experimental area (except the aisles), using a commercial two-row potato planter. Urea-Ammonium sulphate $\left(40-0-0+14 \mathrm{SO}_{3}\right)$ were applied 60 days after planting by hand spreading at a rate of $100 \mathrm{~kg} /$ experimental field. The experimental field was irrigated for 2 hours every other day during the growing season. Irrigation was totally stopped 2 weeks before harvesting.

Metribuzin was applied prior to potato emergence (PRE, $3 \mathrm{WAP}$ ) at $0.35,0.56$ and $0.75 \mathrm{~kg}$ ai/ha. Metribuzin was sprayed by a hand held $\mathrm{CO}_{2}$-pressurized backpack sprayer that delivers $310 \mathrm{~L} / \mathrm{ha}$ at $138 \mathrm{Kpa}$ through a Teejet $8002 \mathrm{flat}$ fan spray tips. Irrigation followed one day after spraying. Hilling was carried out 3 times during the season: 6, 7, and 8 WAP using John Deere rear-mounted, two row ridger with units spaced $90 \mathrm{~cm}$ apart, which formed hills $40 \mathrm{~cm}$ high in the middle row only in each selected plot.

\subsection{Experimental Measurements and Statistical Analyses}

Experimental plots were arranged in a randomised complete block design (RCBD) with four replicates. Blocks were separated by $2.5 \mathrm{~m}$ aisles. Each block was divided into 17 plots, a total of 68 plots/experimental site. The area of each plot was $10.5 \mathrm{~m}^{2}$ ( $5 \mathrm{~m}$ length $\times 2.1 \mathrm{~m}$ width). Each plot consisted of 3 rows, 0.70 $\mathrm{m}$ apart, for a total of 66 rows. Treatments were weedy check, hand weeding, no hilling with different metribuzin rates: $0.35,0.56$, and $0.75 \mathrm{~kg}$ ai/ha, hilling with no metribuzin at different times: 6,7 , and $8 \mathrm{WAP}$, and a combination of the mentioned metribuzin rates and hilling timing. Weed data included weed count $/ \mathrm{m}^{2}$, weed count visual rating (WCVR) on a scale from $0-10$ where 0 is highly infested and 10 no weeds, and weed dry weight. Common weed species found during the growing season in the experimental plots were Amaranthus retroflexus, Convolvulus arvensis, Polygonum aviculare, Portulaca oleracea, Setaria verticillata, Solanum nigrum, Sorghum halepense, and Datura stramonium.

Potato data included number of plants per middle row, height/10 plants/plot, potato roots dry weight of 2 border plants from the middle row, non-marketable and marketable yield (weight and number). Potato yield was determined by harvesting the middle row in each plot using Zahle plough cultivator and then collected by hands. Yield quality was determined by separating harvested tubers into two classes: marketable ( $>6 \mathrm{~cm}$ diameter) and non-marketable tubers $(<5$ $\mathrm{cm}$ in diameter) according to Robinson et al. (1996) [9].

Statistical analyses were performed using STATA (2012). Treatment means were compared using one way ANOVA (analysis of variance) and Tukey's range test. Differences were considered significant at $\alpha=0.05$.

\section{Results and Discussion}

\subsection{Effect on Weed Growth}

Results in Table 1 and Table 2 reveal that metribuzin alone at all tested rates or 
Table 1. Effect of metribuzin, hilling, and their combination on weed count per $\mathrm{m}^{2}$ at 50,70 and 110 days after planting potatoes (DAP). Means followed by the same letter, within each column, do not significantly differ according to Tukey's range test ( $\alpha=$ $0.05)$.

\begin{tabular}{|c|c|c|c|c|c|c|c|c|}
\hline \multirow{3}{*}{$\begin{array}{c}\text { Treatment } \\
\text { Check }\end{array}$} & \multirow{3}{*}{$\begin{array}{c}\text { Rate (kg ai/ha) } \\
0\end{array}$} & \multirow{3}{*}{$\begin{array}{c}\text { Hilling (WAP*) } \\
\text { - }\end{array}$} & \multicolumn{6}{|c|}{ Weed Count $/ \mathrm{m}^{2}$} \\
\hline & & & \multicolumn{2}{|c|}{$50^{* *}$} & \multicolumn{2}{|c|}{70} & \multicolumn{2}{|c|}{110} \\
\hline & & & 476.0 & $\mathrm{~b}$ & 400.0 & $\mathrm{~b}$ & 200.0 & $\mathrm{~b}$ \\
\hline Hand Weeding & 0 & - & 0.0 & a & 0.0 & $\mathrm{a}$ & 0.0 & $\mathrm{a}$ \\
\hline Metribuzin (M) & 0.35 & - & 46.0 & $\mathrm{a}$ & 12.0 & $\mathrm{a}$ & 36.0 & a \\
\hline $\mathbf{M}$ & 0.56 & - & 12.6 & $\mathrm{a}$ & 10.6 & $\mathrm{a}$ & 8.6 & $\mathrm{a}$ \\
\hline $\mathbf{M}$ & 0.75 & - & 23.0 & $\mathrm{a}$ & 41.0 & $\mathrm{a}$ & 40.0 & $\mathrm{a}$ \\
\hline $\mathbf{M}$ & 0 & 6 & 439.6 & $\mathrm{~b}$ & 154.6 & $\mathrm{~b}$ & 160.0 & b \\
\hline $\mathbf{M}$ & 0.35 & 6 & 8.0 & $\mathrm{a}$ & 9.0 & $\mathrm{a}$ & 2.6 & $\mathrm{a}$ \\
\hline $\mathbf{M}$ & 0.56 & 6 & 13.4 & a & 8.0 & a & 5.6 & a \\
\hline $\mathbf{M}$ & 0.75 & 6 & 9.4 & $\mathrm{a}$ & 5.0 & $\mathrm{a}$ & 6.0 & $\mathrm{a}$ \\
\hline $\mathbf{M}$ & 0 & 7 & 581 & $\mathrm{~b}$ & 141 & $\mathrm{~b}$ & 180.0 & b \\
\hline $\mathbf{M}$ & 0.35 & 7 & 48.0 & $\mathrm{a}$ & 30.6 & $\mathrm{a}$ & 11.3 & $\mathrm{a}$ \\
\hline $\mathbf{M}$ & 0.56 & 7 & 4.0 & a & 8.6 & $\mathrm{a}$ & 3.4 & a \\
\hline $\mathbf{M}$ & 0.75 & 7 & 12.0 & $\mathrm{a}$ & 12.0 & $\mathrm{a}$ & 5.4 & a \\
\hline $\mathbf{M}$ & 0 & 8 & 535.6 & $\mathrm{~b}$ & 233.4 & $\mathrm{~b}$ & 193.4 & $b$ \\
\hline $\mathbf{M}$ & 0.35 & 8 & 42.0 & $\mathrm{a}$ & 17.6 & $\mathrm{a}$ & 22.6 & $\mathrm{a}$ \\
\hline $\mathbf{M}$ & 0.56 & 8 & 13.6 & $\mathrm{a}$ & 13.0 & $\mathrm{a}$ & 12.6 & a \\
\hline $\mathbf{M}$ & 0.75 & 8 & 24.0 & a & 4.6 & $\mathrm{a}$ & 8.6 & a \\
\hline
\end{tabular}

${ }^{*}$ WAP: Weeks after planting; ${ }^{* *}$ Means with the same letters in the same column are not significantly different.

Table 2. Effect of metribuzin, hilling, and their combination on weed count visual rating (WCVR) at 50, 70, and 110 days after planting (DAP), and on average weed dry weight. Means followed by the same letter, within each column, do not significantly differ according to Tukey's range test $(\alpha=0.05)$.

\begin{tabular}{|c|c|c|c|c|c|c|c|c|c|c|}
\hline \multirow{3}{*}{$\begin{array}{c}\text { Treatment } \\
\text { Check }\end{array}$} & \multirow{3}{*}{$\begin{array}{c}\text { Rate (kg ai/ha) } \\
0\end{array}$} & \multirow{3}{*}{$\begin{array}{c}\text { Hilling (WAP) } \\
-\end{array}$} & \multicolumn{6}{|c|}{ WCVR } & \multirow{2}{*}{\multicolumn{2}{|c|}{$\frac{\text { Av. weed dry wt } / \mathrm{m}^{2}(\mathrm{~g})}{135}$}} \\
\hline & & & \multicolumn{2}{|c|}{50} & \multicolumn{2}{|c|}{70} & \multicolumn{2}{|c|}{110} & & \\
\hline & & & 3.0 & c & 0.0 & $\mathrm{~b}$ & 2.5 & acd & 789.4 & $\mathrm{e}$ \\
\hline Hand Weeding & 0 & - & 10.0 & $\mathrm{~b}$ & 10.0 & a & 10.0 & $\mathrm{~b}$ & 0.0 & $\mathrm{a}$ \\
\hline Metribuzin (M) & 0.35 & - & 8.3 & $\mathrm{ab}$ & 8.8 & $\mathrm{a}$ & 6.0 & abcd & 62.6 & $\mathrm{a}$ \\
\hline $\mathbf{M}$ & 0.56 & - & 9.3 & $\mathrm{ab}$ & 9.3 & a & 6.5 & abcd & 4.0 & a \\
\hline $\mathbf{M}$ & 0.75 & - & 9.0 & $\mathrm{ab}$ & 7.8 & $\mathrm{a}$ & 5.3 & abcd & 48.0 & a \\
\hline $\mathbf{M}$ & 0 & 6 & 1.5 & c & 3.5 & $\mathrm{~b}$ & 1.0 & $\mathrm{~cd}$ & 342.6 & $\mathrm{~b}$ \\
\hline $\mathbf{M}$ & 0.35 & 6 & 9.0 & $\mathrm{ab}$ & 9.5 & a & 9.8 & $\mathrm{~b}$ & 16.6 & a \\
\hline $\mathbf{M}$ & 0.56 & 6 & 9.1 & $\mathrm{ab}$ & 9.5 & $\mathrm{a}$ & 8.5 & $\mathrm{ab}$ & 38.0 & $\mathrm{a}$ \\
\hline $\mathbf{M}$ & 0.75 & 6 & 9.8 & $\mathrm{ab}$ & 9.8 & $\mathrm{a}$ & 8.0 & $\mathrm{ab}$ & 2.2 & $\mathrm{a}$ \\
\hline $\mathbf{M}$ & 0 & 7 & 0.8 & c & 3.5 & $\mathrm{~b}$ & 0.5 & c & 629.6 & de \\
\hline $\mathbf{M}$ & 0.35 & 7 & 8.3 & $\mathrm{ab}$ & 8.8 & $\mathrm{a}$ & 7.8 & $\mathrm{ab}$ & 38.6 & $\mathrm{a}$ \\
\hline $\mathbf{M}$ & 0.56 & 7 & 9.8 & $a b$ & 9.0 & a & 5.5 & $\mathrm{abcd}$ & 12.6 & a \\
\hline $\mathbf{M}$ & 0.75 & 7 & 9.4 & $a b$ & 8.8 & a & 7.0 & abd & 6.0 & a \\
\hline $\mathbf{M}$ & 0 & 8 & 2.0 & c & 3.3 & b & 3.0 & acd & 654.6 & de \\
\hline $\mathbf{M}$ & 0.35 & 8 & 8.5 & $a b$ & 9.0 & $\mathrm{a}$ & 5.3 & abcd & 92.0 & $a b$ \\
\hline $\mathbf{M}$ & 0.56 & 8 & 9.5 & $a b$ & 10.0 & $\mathrm{a}$ & 7.5 & $\mathrm{ab}$ & 5.4 & $\mathrm{a}$ \\
\hline $\mathbf{M}$ & 0.75 & 8 & 9.5 & $a b$ & 9.3 & $\mathrm{a}$ & 8.5 & $a b$ & 68.6 & $\mathrm{a}$ \\
\hline
\end{tabular}

WCVR scale: 0 - 10; 0 means high weed infestation and 10 means no weeds; WAP: Weeks after planting; means with the same letters in the same column are not significantly different. 
combined with hilling 6,7 , or 8 WAP significantly reduced weed infestation in potato after 50, 70, and 110 DAP compared to the check. Hilling alone 6, 7, and $8 \mathrm{WAP}$ was ineffective against weeds. Combination of metribuzin ( $0.35 \mathrm{~kg}$ ai/ha) and hilling (6 WAP) was the most effective treatment against weeds compared to the check or hilling alone. This treatment gave an excellent control of Amaranthus retroflexus, Chenopodium album, and Sorghum halepense. Combination of metribuzin ( 0.35 and $0.56 \mathrm{~kg}$ ai/ha) and hilling (6 and $7 \mathrm{WAP}$ ) significantly reduced weed infestation compared to hilling alone 6 and 7 WAP at 110 DAP (Table 2).

Hilling at the right time is very critical in weed management. Early hilling can better control weeds than late hilling since weeds are still young and they lack their secondary roots and food reserves. Fully developed weeds will have a sturdy root system that is difficult to destroy; besides these large weeds can block the cultivators upon hilling, or they may pull potato roots relocating them and causing root injury [10]. Our observation shows that early hilling is more effective against weeds than late hilling. Average weed count was 439, 104, and 140 weeds when plots were hilled $6 \mathrm{WAP}$ at 50,70, and 110 DAP, respectively, compared to 581, 122, and 180 weeds when plots were hilled 7 WAP, while it recorded 536, 234, and 193 weeds when plots were hilled 8 WAP (Table 1). Thus, weeds should be controlled at early stages especially when they emerge with the crop since they are more deleterious than those emerging at later crop growth stages. Also, some weeds are difficult to control at later stages due to their morphology such as their deep root systems or underground storage parts as in perennial weeds.

With the exception of hilling alone at 7 and 8 WAP, all treatments significantly reduced the dry weight of weeds compared to the check (Table 2). Average weed dry weights in metribuzin ( $0.75 \mathrm{~kg}$ ai/ha) was $63 \mathrm{~g}$ with no hilling, $17 \mathrm{~g}$ with hilling at $6 \mathrm{WAP}, 39 \mathrm{~g}$ with hilling at $7 \mathrm{WAP}$, and $92 \mathrm{~g}$ with hilling at 8 WAP. Weed dry weights in all the above treatments were significantly lower than the check. But, weed dry weight in hilling alone at 6 WAP was significantly lower than the check in comparison to hilling alone at 7 and 8 WAP. This observation was also reported by [11] [12] [13] [14].

Also, we observed that hilling alone was ineffective against perennial weeds (Unpublished data). This is could be due to their ability to re-germinate promptly from vegetative reproductive structures such as rhizomes or tubers, especially if these structures are dispersed through the field upon cultivation in too wet soil conditions [15]. Moreover, hilling is not effective against weeds within the crop row. Weeds growing early in the season between potato plants are more threatening than those growing between rows. This is because most of them are growing beside the crop and cannot be reached by the hilling machine [16]. In addition, hilling can increase weeds within rows by removing soils from spaces between rows and adding them on the hills between potato plants. This will allow weed seeds buried deep in the soil layers that did not receive any her- 
bicide to be moved to the top of the hills then germinate again in untreated zones [17] after being exposed to light and water [18] [19]. For example, some annual weeds like Setaria spp. and Amaranthus retroflexus are best controlled when hilling is applied at a certain period of the growing term [20].

And since hilling is a time consuming process and requires specific weather conditions, neither too dry nor too wet, this encouraged farmers to find an alternative weed control strategy [21]. Currently, many farmers are relying on herbicides while reducing or excluding hilling practices in order to manage weeds [22]. In potato fields, pre-emergence or post-emergence herbicides can be used to control weeds. Most grasses and some broadleaved weeds, such as Xanthium strumarium, Capsella bursa-pastoris, and Sinapis arvensis are better controlled by pre-emergence herbicides ([23]. Our data suggest that, for best weed management, metribuzin use in potato could be reduced by $53 \%$ with hilling at 6 or 7 WAP.

\subsection{Effect on Potato Growth and Development}

Hilling or metribuzin and their combinations had no negative effect on potato plants. Average shoot height (Table 3), plant number, shoot number, leaf number (Table 4), and average root dry weights (Table 5) showed no significant differences among all treatments compared to the check, at 50, 70, and 110 DAP.

Table 3. Effect of metribuzin, hilling, and their combination on average potato height $(\mathrm{cm})$ per 10 plants per middle row at 50 , 70 , and 110 days after planting (DAP). Means followed by the same letter, within each column, do not significantly differ according to Tukey's range test $(\alpha=0.05)$.

\begin{tabular}{|c|c|c|c|c|c|c|c|c|}
\hline \multirow{3}{*}{$\begin{array}{c}\text { Treatment } \\
\text { Check }\end{array}$} & \multirow{3}{*}{$\begin{array}{c}\text { Rate (kg ai/ha) } \\
0\end{array}$} & \multirow{3}{*}{$\begin{array}{c}\text { Hilling (WAP) } \\
-\end{array}$} & \multicolumn{6}{|c|}{ Average potato height $(\mathrm{cm}) / 10$ plants DAP } \\
\hline & & & \multicolumn{2}{|c|}{50} & \multicolumn{2}{|c|}{70} & \multicolumn{2}{|c|}{110} \\
\hline & & & 35.0 & $\mathrm{ab}$ & 59.4 & a & 74.6 & $\mathrm{ab}$ \\
\hline Hand Weeding & 0 & - & 33.5 & $\mathrm{ab}$ & 65.5 & a & 66.0 & $\mathrm{~b}$ \\
\hline Metrbuzin (M) & 0.35 & - & 31.4 & $\mathrm{ab}$ & 70.6 & a & 90.5 & $a b$ \\
\hline $\mathbf{M}$ & 0.56 & - & 27.0 & $\mathrm{ab}$ & 69.7 & a & 102.2 & a \\
\hline M & 0.75 & - & 21.5 & a & 67.3 & a & 99.4 & $\mathrm{ab}$ \\
\hline M & 0 & 6 & 31.8 & $a b$ & 63.5 & $\mathrm{a}$ & 93.0 & $a b$ \\
\hline $\mathbf{M}$ & 0.35 & 6 & 33.0 & $\mathrm{ab}$ & 70.7 & $\mathrm{a}$ & 102.8 & $\mathrm{a}$ \\
\hline $\mathbf{M}$ & 0.56 & 6 & 26.2 & $\mathrm{ab}$ & 54.5 & a & 107.2 & $\mathrm{a}$ \\
\hline $\mathbf{M}$ & 0.75 & 6 & 28.4 & $\mathrm{ab}$ & 68.4 & $\mathrm{a}$ & 106.7 & $\mathrm{a}$ \\
\hline $\mathbf{M}$ & 0 & 7 & 37.8 & $\mathrm{~b}$ & 59.8 & $\mathrm{a}$ & 75.1 & $\mathrm{ab}$ \\
\hline $\mathbf{M}$ & 0.35 & 7 & 30.9 & $\mathrm{ab}$ & 72.0 & $\mathrm{a}$ & 101.2 & $\mathrm{a}$ \\
\hline $\mathbf{M}$ & 0.56 & 7 & 29.9 & $\mathrm{ab}$ & 65.0 & $\mathrm{a}$ & 86.0 & $a b$ \\
\hline $\mathbf{M}$ & 0.75 & 7 & 29.3 & $\mathrm{ab}$ & 70.0 & $\mathrm{a}$ & 101.2 & $\mathrm{a}$ \\
\hline $\mathbf{M}$ & 0 & 8 & 37.2 & $\mathrm{ab}$ & 62.3 & $\mathrm{a}$ & 80.3 & $\mathrm{ab}$ \\
\hline $\mathbf{M}$ & 0.35 & 8 & 31.4 & $\mathrm{ab}$ & 69.0 & $\mathrm{a}$ & 100.0 & $\mathrm{ab}$ \\
\hline $\mathbf{M}$ & 0.56 & 8 & 29.4 & $\mathrm{ab}$ & 65.6 & $\mathrm{a}$ & 93.8 & $a b$ \\
\hline $\mathbf{M}$ & 0.75 & 8 & 31.4 & $\mathrm{ab}$ & 68.9 & $\mathrm{a}$ & 95.0 & $a b$ \\
\hline
\end{tabular}

WAP: Weeks after planting; means with the same letters in the same column are not significantly different. 
Table 4. Effect of metribuzin, hilling, and their combination on average potato plant number per plant middle row, 50 days after planting (DAP), average shoot number per plant, 50 and 70 DAP, and on average leaf number per plant, 110 DAP. Means followed by the same letter, within each column, do not significantly differ according to Tukey's range test $(\alpha=0.05)$.

\begin{tabular}{|c|c|c|c|c|c|c|c|c|c|c|}
\hline \multirow{3}{*}{$\begin{array}{c}\text { Treatment } \\
\text { Check }\end{array}$} & \multirow{3}{*}{$\begin{array}{c}\text { Rate (kg ai/ha) } \\
0\end{array}$} & \multirow{3}{*}{$\begin{array}{c}\text { Hilling (WAP) } \\
-\end{array}$} & \multirow{2}{*}{\multicolumn{2}{|c|}{$\begin{array}{c}\text { Pot. \#/middle row } \\
50\end{array}$}} & \multicolumn{4}{|c|}{ Average shoot \#/plant } & \multirow{2}{*}{\multicolumn{2}{|c|}{$\begin{array}{c}\text { Average leaf \#/plant } \\
110\end{array}$}} \\
\hline & & & & & \multicolumn{2}{|c|}{50} & \multicolumn{2}{|c|}{70} & & \\
\hline & & & 18.0 & a & 2.6 & a & 1.8 & a & 23.1 & $\mathrm{a}$ \\
\hline Hand Weeding & 0 & - & 19.0 & $\mathrm{a}$ & 2.9 & $\mathrm{a}$ & 2.5 & $\mathrm{a}$ & 46.2 & $\mathrm{a}$ \\
\hline Metribuzin (M) & 0.35 & - & 16.5 & a & 2.2 & a & 2.0 & a & 57.7 & a \\
\hline $\mathbf{M}$ & 0.56 & - & 18.0 & a & 2.3 & a & 2.3 & a & 51.3 & a \\
\hline M & 0.75 & - & 16.8 & $\mathrm{a}$ & 2.2 & a & 2.2 & $\mathrm{a}$ & 40.7 & $\mathrm{a}$ \\
\hline $\mathbf{M}$ & 0 & 6 & 17.3 & a & 2.4 & a & 2.1 & $\mathrm{a}$ & 36.4 & a \\
\hline $\mathbf{M}$ & 0.35 & 6 & 18.0 & $\mathrm{a}$ & 2.6 & a & 2.1 & a & 61.8 & a \\
\hline $\mathbf{M}$ & 0.56 & 6 & 17.0 & a & 2.2 & a & 2.3 & a & 55.9 & a \\
\hline $\mathbf{M}$ & 0.75 & 6 & 16.8 & $\mathrm{a}$ & 2.4 & $\mathrm{a}$ & 2.3 & $\mathrm{a}$ & 72.7 & $\mathrm{a}$ \\
\hline $\mathbf{M}$ & 0 & 7 & 17.5 & $\mathrm{a}$ & 3.0 & a & 2.7 & $\mathrm{a}$ & 42.1 & a \\
\hline $\mathbf{M}$ & 0.35 & 7 & 18.3 & $\mathrm{a}$ & 2.4 & $\mathrm{a}$ & 2.3 & $\mathrm{a}$ & 63.0 & $\mathrm{a}$ \\
\hline $\mathbf{M}$ & 0.56 & 7 & 16.5 & $\mathrm{a}$ & 2.2 & $\mathrm{a}$ & 1.9 & $\mathrm{a}$ & 55.9 & $\mathrm{a}$ \\
\hline $\mathbf{M}$ & 0.75 & 7 & 17.0 & a & 2.5 & a & 2.3 & $\mathrm{a}$ & 52.7 & a \\
\hline $\mathbf{M}$ & 0 & 8 & 18.3 & $\mathrm{a}$ & 3.1 & a & 2.7 & $\mathrm{a}$ & 37.0 & $\mathrm{a}$ \\
\hline $\mathbf{M}$ & 0.35 & 8 & 17.3 & $\mathrm{a}$ & 2.6 & $\mathrm{a}$ & 2.2 & $\mathrm{a}$ & 54.0 & $\mathrm{a}$ \\
\hline $\mathbf{M}$ & 0.56 & 8 & 18.3 & a & 2.3 & $\mathrm{a}$ & 2.2 & $\mathrm{a}$ & 56.6 & $\mathrm{a}$ \\
\hline $\mathbf{M}$ & 0.75 & 8 & 16.8 & $\mathrm{a}$ & 2.3 & $\mathrm{a}$ & 2.7 & $\mathrm{a}$ & 45.5 & $\mathrm{a}$ \\
\hline
\end{tabular}

WAP: Weeks after planting; means with the same letters in the same column are not significantly different.

Table 5. Effect of metribuzin, hilling, and their combination on average potato shoot number and root dry weight (g) per two plants, 70 days after planting (DAP). Means followed by the same letter, within each column, do not significantly differ according to Tukey's range test $(\alpha=0.05)$.

\begin{tabular}{|c|c|c|c|c|}
\hline \multirow{2}{*}{$\begin{array}{c}\text { Treatment } \\
\text { Check }\end{array}$} & \multirow{2}{*}{$\begin{array}{c}\text { Rate (kg ai/ha) } \\
0\end{array}$} & \multirow{2}{*}{$\begin{array}{c}\text { Hilling (WAP) } \\
-\end{array}$} & \multicolumn{2}{|c|}{ Average root dry weight (g) } \\
\hline & & & 9.3 & a \\
\hline Hand Weeding & 0 & - & 12.9 & a \\
\hline Metribuzin (M) & 0.35 & - & 9.6 & a \\
\hline $\mathbf{M}$ & 0.56 & - & 12.3 & a \\
\hline $\mathbf{M}$ & 0.75 & - & 11.1 & a \\
\hline $\mathbf{M}$ & 0 & 6 & 12.3 & a \\
\hline $\mathbf{M}$ & 0.35 & 6 & 11.7 & a \\
\hline $\mathbf{M}$ & 0.56 & 6 & 12.1 & a \\
\hline $\mathbf{M}$ & 0.75 & 6 & 13.4 & a \\
\hline $\mathbf{M}$ & 0 & 7 & 9.2 & a \\
\hline $\mathbf{M}$ & 0.35 & 7 & 11.0 & a \\
\hline $\mathbf{M}$ & 0.56 & 7 & 12.9 & a \\
\hline $\mathbf{M}$ & 0.75 & 7 & 11.7 & a \\
\hline $\mathbf{M}$ & 0 & 8 & 10.8 & a \\
\hline $\mathbf{M}$ & 0.35 & 8 & 13.6 & $\mathrm{a}$ \\
\hline $\mathbf{M}$ & 0.56 & 8 & 12.1 & $\mathrm{a}$ \\
\hline $\mathbf{M}$ & 0.75 & 8 & 13.1 & $\mathrm{a}$ \\
\hline
\end{tabular}

WAP: Weeks after planting; means with the same letters in the same column are not significantly different. 
Table 6 shows that except for hilling alone at 6, 7, and 8 WAP, metribuzin alone at all tested rates, with or without hilling, significantly increased marketable potato tuber weight and total tuber yield, in comparison to the check. The highest marketable potato yield (36.49 tons/ha) was observed with metribuzin at $0.36 \mathrm{~kg}$ ai/ha with hilling $6 \mathrm{WAP}$. This treatment was better than hand weeded plots (29.51 tons/ha of marketable tubers). In addition, it was observed that marketable yield in plots hilled at $6 \mathrm{WAP}$ is higher than plots hilled later in the season (7 and $8 \mathrm{WAP}$ ), with an average of 15.45 tons/ha, compared to 6.64 and 8.53 tons/ha in plots hilled 7 and 8 WAP, respectively.

Same results were observed regarding potato tuber numbers (Table 7). There were 58,572 marketable tubers in plots hilled $6 \mathrm{WAP}$, compared to 27,143 marketable tubers in plots hilled 7 WAP, and 35,000 marketable tubers in plots hilled 8 WAP with no metribuzin, even though there were no significance differences among them in comparison to the check. Regarding metribuzin treatments, marketable and non-marketable tuber numbers showed no significant difference among all treatments of metribuzin, with or without hilling, but significantly different compared to the check and to all hilled plots. Weed control at

Table 6. Effect of metribuzin, hilling, and their combination on average marketable, non-marketable, and total potato tuber weight (tons/ha), 130 days after planting (DAP). Means followed by the same letter, within each column, do not significantly differ according to Tukey's range test $(\alpha=0.05)$.

\begin{tabular}{|c|c|c|c|c|c|c|c|c|}
\hline \multirow{3}{*}{$\begin{array}{c}\text { Treatment } \\
\text { Check }\end{array}$} & \multirow{3}{*}{$\begin{array}{c}\text { Rate (kg ai/ha) } \\
0\end{array}$} & \multirow{3}{*}{$\begin{array}{c}\text { Hilling (WAP) } \\
-\end{array}$} & \multicolumn{6}{|c|}{ Average potato tuber weight $(\mathrm{t} / \mathrm{ha})$} \\
\hline & & & \multicolumn{2}{|c|}{ Marketable } & \multicolumn{2}{|c|}{ Non-marketable } & \multicolumn{2}{|c|}{ Total } \\
\hline & & & 3.46 & e & 14.81 & a & 18.27 & $\mathrm{e}$ \\
\hline Hand Weeding & 0 & - & 29.51 & abcd & 26.38 & $\mathrm{~b}$ & 55.89 & $\mathrm{ab}$ \\
\hline Metribuzin (M) & 0.35 & - & 29.85 & $a b c$ & 18.80 & $\mathrm{ab}$ & 48.65 & abcd \\
\hline $\mathbf{M}$ & 0.56 & - & 31.32 & $a b c$ & 16.76 & $\mathrm{ab}$ & 48.08 & abcd \\
\hline $\mathbf{M}$ & 0.75 & - & 31.42 & $a b c$ & 14.48 & $\mathrm{a}$ & 45.90 & abcd \\
\hline $\mathbf{M}$ & 0 & 6 & 15.45 & acde & 14.67 & a & 30.12 & acde \\
\hline $\mathbf{M}$ & 0.35 & 6 & 36.49 & $\mathrm{ab}$ & 16.47 & $a b$ & 52.97 & $a b c$ \\
\hline $\mathbf{M}$ & 0.56 & 6 & 34.37 & $a b$ & 18.06 & $a b$ & 52.43 & $a b$ \\
\hline $\mathbf{M}$ & 0.75 & 6 & 36.00 & $\mathrm{ab}$ & 15.43 & $a b$ & 51.43 & $\mathrm{ab}$ \\
\hline $\mathbf{M}$ & 0 & 7 & 6.64 & de & 16.25 & $a b$ & 22.88 & de \\
\hline $\mathbf{M}$ & 0.35 & 7 & 27.85 & $a b c d$ & 18.75 & $a b$ & 46.60 & abcd \\
\hline $\mathbf{M}$ & 0.56 & 7 & 27.09 & $a b c d$ & 16.46 & $a b$ & 43.55 & abcde \\
\hline $\mathbf{M}$ & 0.75 & 7 & 34.73 & $\mathrm{ab}$ & 20.18 & $a b$ & 54.91 & $a b$ \\
\hline $\mathbf{M}$ & 0 & 8 & 8.53 & cde & 16.61 & $a b$ & 25.14 & cde \\
\hline $\mathbf{M}$ & 0.35 & 8 & 30.65 & $a b c$ & 18.34 & $a b$ & 48.98 & $a b c$ \\
\hline $\mathbf{M}$ & 0.56 & 8 & 36.32 & $a b$ & 18.52 & $a b$ & 55.84 & $a b$ \\
\hline $\mathbf{M}$ & 0.75 & 8 & 30.67 & $a b c$ & 21.30 & $\mathrm{ab}$ & 51.97 & $a b$ \\
\hline
\end{tabular}

WAP: Weeks after planting; means with the same letters in the same column are not significantly different. 
Table 7. Effect of metribuzin, hilling, and their combination on marketable, non-marketable, and total potato tuber numbers (tubers/ha), 130 days after planting (DAP). Means followed by the same letter, within each column, do not significantly differ according to Tukey's range test $(\alpha=0.05)$.

\begin{tabular}{|c|c|c|c|c|c|c|c|c|}
\hline \multirow{3}{*}{$\begin{array}{c}\text { Treatment } \\
\text { Check }\end{array}$} & \multirow{3}{*}{$\begin{array}{c}\text { Rate (kg ai/ha) } \\
0\end{array}$} & \multirow{3}{*}{$\begin{array}{l}\text { Hilling (WAP) } \\
\text { - }\end{array}$} & \multicolumn{6}{|c|}{ Average potato tuber number (tubers/ha) } \\
\hline & & & \multicolumn{2}{|c|}{ Marketable } & \multicolumn{2}{|c|}{ Non-marketable } & \multicolumn{2}{|c|}{ Total } \\
\hline & & & 13,572 & $\mathrm{~d}$ & 172,143 & a & 185,714 & $\mathrm{a}$ \\
\hline Hand Weeding & 0 & - & 98,571 & $\mathrm{ab}$ & 237,857 & a & 336,429 & $\mathrm{~b}$ \\
\hline Metribuzin (M) & 0.35 & - & 96,429 & $\mathrm{ab}$ & 178,571 & a & 275,000 & $\mathrm{ab}$ \\
\hline $\mathbf{M}$ & 0.56 & - & 116,429 & a & 150,714 & a & 267,143 & $\mathrm{ab}$ \\
\hline $\mathbf{M}$ & 0.75 & - & 82,857 & $a b c$ & 131,429 & a & 214,286 & $\mathrm{ab}$ \\
\hline $\mathbf{M}$ & 0 & 6 & 58,572 & abcd & 152,143 & a & 210,714 & $\mathrm{ab}$ \\
\hline $\mathbf{M}$ & 0.35 & 6 & 104,286 & $\mathrm{a}$ & 191,429 & a & 295,714 & $a b$ \\
\hline $\mathbf{M}$ & 0.56 & 6 & 106,429 & a & 180,714 & $\mathrm{a}$ & 287,143 & $a b$ \\
\hline $\mathbf{M}$ & 0.75 & 6 & 106,429 & $\mathrm{a}$ & 147,857 & $\mathrm{a}$ & 254,286 & $a b$ \\
\hline $\mathbf{M}$ & 0 & 7 & 27,143 & $c d$ & 167,857 & $\mathrm{a}$ & 195,000 & $\mathrm{ab}$ \\
\hline $\mathbf{M}$ & 0.35 & 7 & 70,715 & abcd & 185,714 & a & 256,429 & $a b$ \\
\hline $\mathbf{M}$ & 0.56 & 7 & 89,286 & $a b c$ & 170,714 & $\mathrm{a}$ & 260,000 & $a b$ \\
\hline $\mathbf{M}$ & 0.75 & 7 & 108,572 & a & 188,571 & a & 297,143 & $\mathrm{ab}$ \\
\hline $\mathbf{M}$ & 0 & 8 & 35,000 & bcd & 195,714 & $\mathrm{a}$ & 230,714 & $a b$ \\
\hline $\mathbf{M}$ & 0.35 & 8 & 96,429 & $\mathrm{ab}$ & 182,143 & a & 278,571 & $\mathrm{ab}$ \\
\hline $\mathbf{M}$ & 0.56 & 8 & 126,428 & $\mathrm{a}$ & 157,857 & $\mathrm{a}$ & 284,286 & $a b$ \\
\hline $\mathbf{M}$ & 0.75 & 8 & 94,286 & $a b c$ & 170,714 & $\mathrm{a}$ & 265,000 & $a b$ \\
\hline
\end{tabular}

WAP: Weeks after planting; means with the same letters in the same column are not significantly different.

the proper timing can increase potato yield by 15\% - 50\% [7] [11]. Thus, hilling time should be considered as a significant factor to maintain good potato yields early in the season and to reduce the effect of weeds emerging later [25]. In addition, early hilling could aid in covering the tubers from sunlight, reducing culls, aerating soil [2] and facilitating harvesting since less soil-at least $40 \%$ less-is present in the space between potato rows where cultivators pass [26]. However, late hilling may cause pruning of potato roots and stolons, breaking soil structure, compacting soil, or causing erosion if heavy machinery were implemented or if several cultivations were applied [17] [27]. Using reduced rate of metribuzin before hilling was successful in controlling weeds and enhancing potato yield. The results indicate that low rates of metribuzin applied before hilling at 6 WAP will provide optimum weed control in potato.

\section{Conclusion}

All tested rates of metribuzin were effective against weeds, while hilling alone 6, 7 , and 8 WAP was ineffective against weeds. Any rate of metribuzin, combined with any hilling time, was effective against weeds and can greatly enhance long 
season weed control during that season. All treatments had no negative effect on potato plants or tuber yields. Additional research is recommended under different locations in Lebanon before a final recommendation to potato growers for the use of low rates of metribuzin can be made.

\section{References}

[1] Abou-Jawdah, Y., Sobh, H. and Saad, A. (2001) Incidence of Potato Virus Diseases and Their Significance for a Seed Certification Program in Lebanon. Phytopathologia Mediterranea, 40, 113-118.

[2] Bellinder, R., Wallace, R. and Wilkins, E. (1996) Reduced Rates of Herbicides Following Hilling Controlled Weeds in Conventional and Reduced Tillage Potato (Solanum tuberosum) Production. Weed Technology, 10, 311-316.

[3] Binning, L., Connell, T., Kienitz, J., Michaelis, B. and Hughes, R. (1991) Movement and Dissipation of Three Potato Herbicides. American Potato Journal, 68, 597-597.

[4] Burgard, D., Dowdy, R., Koskinen, W. and Cheng, W. (1994) Movement of Metribuzin in a Loamy Sand Soil under Irrigated Potato Production. Weed Science, 42, 446-452.

[5] Chitsaz, M. and Nelson, D. (1983) Comparison of Various Weed Control Programs for Potatoes. American Potato Journal, 60, 271-280. https://doi.org/10.1007/BF02854277

[6] Sieczka, J. and Creighton, J. (1984) Weed Control of Potatoes on Long Island. Proceedings of Northeastern Weed Science Society, 39, 176-180.

[7] Eberlein, C., Patterson, P., Guttieri, M. and Stark, J. (1997) Efficacy and Economics of Cultivation for Weed Control in Potato (Solanum tuberosum). Weed Technology, 11, 257-264.

[8] Bashour, I. and Sayegh, A. (2007) Methods of Analysis for Soils of Arid and Semi-Arid Regions. FAO, Rome.

[9] Robinson, D., Monks, D. and Monaco, J. (1996) Potato (Solanum tuberosum) Tolerance and Susceptibility of Eight Weeds to Rimsulfuron with and without Metribuzin. Weed Technology, 10, 29-34.

[10] Felix, J., Ivany, J., Kegode, G. and Doohan, D. (2009) Timing Potato Cultivation Using the Weed Cast Model. Weed Science, 57, 87-93. https://doi.org/10.1614/WS-08-019.1

[11] Jaiswal, V. and Lal, S. (1996) Efficacy of Cultural and Chemical Weed Control Methods in Potato (Solanum tuberosum). Indian Journal of Agronomy, 41, 454-456.

[12] Suryanarayana Reddy, V. (1993) Chemical Weed Control in Potato (Solanum tuberosum L.) under Irrigated Conditions. Master's Thesis, University of Agricultural Sciences, Bangalore, India.

[13] Singh, K. (1992) Weed Management in Potato (Solanum tuberosum L.) Crop Growth in Acidic Hill Soils of Meghalaya. Indian Journal of Agronomy, 37, 613-614.

[14] Maliwal, P. and Jain, L. (1991) Efficacy of Fluchloralin and Methabenzthiazuran for Selective Weed Control in Potato. Indian Journal of Agronomy, 36, 258-260.

[15] Gianessi, L. and Sankula, S. (2003) The Value of Herbicides in U.S. Crop Production. National Center for Food and Agricultural Policy, Washington DC.

[16] Yip, C., Sweet, R. and Sieczka, J. (1974) Competitive Ability of Potato Cultivars with Major Weed Species. Northeastern Weed Science Society Proceedings, 28, 271-281. 
[17] Rioux, R., Comeau, J. and Genereux, H. (1979) Effect of Cultural Practices and Herbicides on Weed Population and Competition in Potatoes. Canadian Journal Plant Science, 59, 367-374. https://doi.org/10.4141/cjps79-059

[18] Lanfranconi, L., Bellinder, R. and Wallace, R. (1993) Grain Rye Residues and Weed Control Strategies in Reduced Tillage Potatoes. Weed Technology, 7, 23-28.

[19] VanGessel, M. and Renner, K. (1990) Redroot Pigweed (Amaranthus retroflexus) and Barnyard Grass (Echinochloa crus-galli) Interference in Potatoes (Solanum tuberosum). Weed Science, 38, 539-542.

[20] Nalewaja, J., Dexter, A., Buchli, J., Hamlin, W. and Kimmet, G. (1980) Pesticide Usage in Major North Dakota Crops, 1978. Agronomy Report 1. North Dakota State University, Fargo.

[21] Rikoon, J., Vicker, R. and Constance, D. (1993) Factors Affecting Initial Use and Decisions to Abandon Banded Pesticide Applications. In: Agricultural Research to Protect Water Quality Proceedings, Soil and Water Conservation Society, Ankeny, IA, 335-337.

[22] Schweizer, E., Zimdahl, R. and Mickelson, R. (1989) Weed Control in Corn (Zea mays) as Affected by Till-Plant Systems and Herbicides. Weed Science, 3, 162-165.

[23] Hutchinson, P. (2012) Weed Control and Potato Crop Safety with Metribuzin. University of Idaho Extension CIS 1185, University of Idaho, Moscow, Idaho, USA. http://www.cals.uidaho.edu/edcomm/pdf/CIS/CIS1185.pdf

[24] Jaiswal, V. (1994) Different Response of Weed Species to Herbicides in Potato. Journal of Indian Potato Association, 21, 157-159.

[25] Nelson, D. and Thoreson, M. (1981) Competition between Potatoes (Solanum tuberosum) and Weeds. Weed Science, 29, 672-677.

[26] Han, G. and Qi, H. (2013) The Economic Value of a New Technology in Growing Potatoes: The Hilling Method. International Journal of Business \& Economics Perspectives, 8, 53-61.

[27] Nelson, D. and Giles, J. (1986) Abstract. Implication of Post Emergence Tillage on Root Injury and Yields of Potatoes. American Potato Journal, 63, 445. 